\title{
Assessment of Renalase Activity on Catecholamines Degradation
}

\author{
Janete Quelhas-Santos ${ }^{1, *}$, Benedita Sampaio-Maia ${ }^{2}$, Fernando Remião ${ }^{3}$, Paula Serrão, \\ Isabel Soares-Silva ${ }^{1}$, Gary V. Desir ${ }^{5}$ and Manuel Pestana ${ }^{1,6}$
}

\begin{abstract}
${ }^{1}$ Instituto de Investigação e Inovação em Saúde, Universidade do Porto, Portugal \& Nephrology and Infectious Diseases Research and Development Group, INEB, University of Porto, Alameda Prof. Hernâni Monteiro, 4200-319, Porto, Portugal; ${ }^{2}$ Faculty of Dental Medicine, University of Porto, Rua Dr. Manuel Pereira da Silva, 4200-392 Porto, Portugal; ${ }^{3}$ REQUIMTE, Laboratório de Toxicologia, Departamento de Ciências Biológicas, Faculdade de Farmácia, Universidade do Porto, Rua de Jorge Viterbo Ferreira, $n^{\circ} 228$, 4050-313 Porto, Portugal; ${ }^{4}$ Department of Pharmacology and Therapeutics, Faculty of Medicine, University of Porto, Alameda Prof. Hernâni Monteiro, 4200-319, Porto, Portugal; ${ }^{5}$ Yale University School of Medicine, Department of Medicine, New Haven, \& VACHS Medical Center, West Haven, CT, USA; ${ }^{6}$ Hospital de S. João EPE, Alameda Prof. Hernâni Monteiro, 4200-319, Porto, Portugal
\end{abstract}

\begin{abstract}
Renalase was recently described as a new flavoprotein that functions as FAD/NADH-dependent oxidase and, in contrast to other monoamine oxidases, is secreted into plasma and urine. Recombinant renalase was found to exert powerful and rapid hypotensive effects when administered intravenously on rats and this was suggested to be mediated by circulating catecholamines degradation. However there is no concrete evidence that directly supports the hypothesis that renalase metabolizes catecholamines. In this study we aimed to evaluate the catecholamines-degrading renalase activity by three different technical approaches: 1) Amplex Red Monoamine Oxidase Assay, which evaluates the rate of resaruzin reduction by renalase oxidative activity; 2) assessment of catecholamines consumptions by high-pressure liquid chromatography (HPLC) with electrochemical-detection (ED) and 3) assessment of product formation by HPLC with photodiode array-detection (DAD). Using the Amplex Red MAO Assay, all three catecholamines were degraded by recombinant renalase overtime, being adrenaline the preferred substrate, followed by noradrenaline and dopamine. In addition using HPLC-ED, it was observed the consumption of all three catecholamines by recombinant renalase, which were oxidized to the correspondent aminochromes, as observed by DAD. However the role of renalase as catalyzer of aminochromes production is still undefined. In summary, the data presented in this study propose by different methodologies the involvement of renalase in catecholamine metabolization.
\end{abstract}

Keywords: Aminochromes, catecholamines, monoamine oxidase activity, renalase.

\section{INTRODUCTION}

Renalase was recently described as a new flavoprotein, that functions as FAD-dependent oxidase and, in contrast to other monoamine oxidases, is secreted into plasma and urine where it was suggested to metabolize catecholamines [1, 2]. In their first study, $\mathrm{Xu}$ et al. reported that both the recombinant GST-renalase fusion and the natural human protein isolated from urine possessed amine oxidase, but presented a distinct substrate specificity and inhibitor profile of the monoamine-oxidase type A (MAO-A) and type B (MAO-B) [1]. The amine oxidase activity was specifically inhibited by a renalase antibody [1], supporting the notion that the rate of resaruzin reduction measured by the Amplex Red Monoamine Oxidase Assay Kit could be used as an indirect measure of renalase oxidase activity [3,4]. These findings were strongly questioned by Boomsma and Tipton [5], who pointed out that the rate of hydrogen peroxide $\left(\mathrm{H}_{2} \mathrm{O}_{2}\right)$ generation, determined by this method, was far too low to be

\footnotetext{
*Address correspondence to this author at the Nephrology and Infectious Diseases Research and Development Group, INEB \& Hospital de S. João EPE, University of Porto, Alameda Prof. Hernâni Monteiro, 4200-319, Porto, Portugal; Tel: 351-22-5512100; Fax: 351-22-5512228;

E-mail: sjanete@med.up.pt
}

ascribed to enzymatic conversion of catecholamines by renalase [5]. However, it was later reported that NADH was required as a cofactor for the enzymatic function of renalase $[6,7]$, and that incubating recombinant renalase with 250 $\mu \mathrm{M}$ of $\mathrm{NADH}$ the rate of epinephrine metabolism increased by $\sim 18$-fold $[8,9]$. Based on these findings it has been suggested that renalase act as a catecholamine-degrading enzyme, via either $\mathrm{O}_{2}$-dependent or NADH-dependent mechanisms, and recently it was suggested as possible metabolic end-products aminochromes [10].

In in vivo studies, recombinant renalase was found to exert powerful and rapid hypotensive effects when administered intravenously on rats and this was suggested to be mediated by circulating catecholamines degradation $[1,2]$. Recently, renalase was suggested to metabolize preferentially circulating epinephrine and its capacity to decrease blood pressure is directly correlated to its enzymatic activity [4]. However, to metabolize epinephrine, renalase uses a mechanism that is different than that used by the MAOs. Indeed, unlike MAOs, renalase uses NADH as a cofactor and reacts with oxygen to generate superoxide anion. This reaction scheme is similar to that of flavoprotein monooxygenases, a diverse class of enzymes that can catalyze a wide variety of oxidation reactions, including amine oxidation. 
By contrast, Aliverti's group reported that their recombinant FAD-containing human renalase was completely devoid of any amine oxidase activity, although eliciting the expected effects on blood pressure when injected into rats [11]. These authors concluded that the functional properties of their recombinant renalase strongly suggest that it is not a $\mathrm{NAD}(\mathrm{P}) \mathrm{H}-$ dependent enzyme, that it is not a MAO-like enzyme and likely it is not even an oxidase, given that the reactivity of the FAD prosthetic group of renalase dramatically differs from that of MAO [6]. These observations were refuted by claiming that Aliverti's group did not detect renalase's amine oxidase activity because NADH was not included in the assays [3].

Others authors also raised serious doubts regarding the putative catecholamine-degrading activity of renalase suggesting that although renalase may have an important physiological role in the context of hypertension and cardiorenal disease, this appears unlikely to be mediated by degradation of catecholamines [3].

More recently, Moran's group found that in fact renalase does bind to epinephrine, but performs no cataltyic transformation of it [12]. The authors discovered that renalase functions as an $\alpha-\mathrm{NAD}(\mathrm{P}) \mathrm{H}$ oxidase/anomerase, whereby the substrate $\alpha$-dihydropyridyl ring is oxidized by transferring two electrons to the flavin bound cofactor, and the configuration of the ribose $\mathrm{C} 1$ is converted from $\alpha$ to $\beta$. The reduced FAD cofactor then reoxidizes by reacting with dioxygen to yield $\mathrm{H}_{2} \mathrm{O}_{2}$ [12]. They claimed that this reaction is novel in that is the first $\alpha-\mathrm{NAD}(\mathrm{P}) \mathrm{H}$ specific enzyme found in humans [12]. It's not surprising that it is found in many tissues (heart, kidney, brain) due to the ubiquity of $\mathrm{NAD}(\mathrm{P}) \mathrm{H}$ and the need to recycle any alpha as the body has no other known use for this anonomer. One by-product of this reaction is $\mathrm{H}_{2} \mathrm{O}_{2}$, which has been known to cause vasodilation and could have contributed to the previously observed hypotension in rat trials.

Because in the literature, there is no concrete evidence that directly supports the hypothesis that renalase degrades catecholamines, with exception for the catecholaminemetabolising activity based on the production of $\mathrm{H}_{2} \mathrm{O}_{2}$ evaluated by the Amplex Red Monoamine Oxidase Assay during incubation of renalase with catecholamines, the development of a standardized validated method for assessing renalase activity in various fluids is a priority.

In this study, we tested alternative methods to evaluate the catecholamines-degrading renalase activity, namely by evaluating substrate consumption and end-product formation by HPLC.

\section{MATERIALS AND METHODS}

\section{Amplex Red Monoamine Oxidase Kit}

Amine oxidase activity of recombinant renalase was measured using an Amplex Red Monoamine Oxidase Assay Kit (Invitrogen, Life Technologies Ltd, Paisley, UK) [1, 13]. The assay is based on the detection of $\mathrm{H}_{2} \mathrm{O}_{2}$ in a horseradish peroxidase-coupled reaction using 10 acetyl-3,7-dihydroxyphenozazine (Amplex Red reagent Resaruzin, Invitrogen).
The Amplex Red reagent reacts with $\mathrm{H}_{2} \mathrm{O}_{2}$ in a $1: 1$ stoichiometry, generating a fluorescente metabolite resorufin. The fluorescence signal is directly proportional to $\mathrm{H}_{2} \mathrm{O}_{2}$ production and hence amine oxidase enzymatic activity. Experiments were carried out according to the manufacturer's instructions with a final catecholamine (adrenaline, noradrenaline and dopamine) concentration of $2 \mathrm{mM}$, using semicarbazide $(0.5 \mu \mathrm{M})$ as a specific inhibitor of semicarbazide-sensitive amine oxidase (SSAO) in addition to clorgyline and pargyline, as specific inhibitors of MAO-A and $\mathrm{B}$, as well as NADH as a cofactor $(250 \mu \mathrm{M})$ and of recombinant renalase $(100 \mu \mathrm{g} / \mathrm{mL})$. The amount of resorufin generated was determined using a standard curve using resorufin concentrations from 0 to $20 \mu \mathrm{M}$. Fluorescence intensity was measured during 30 minutes, using a fluorescent plate reader (excitation at $530 \mathrm{~nm}$, emission detection at $590 \mathrm{~nm}$ ).

\section{HPLC with Electrochemical Detection (HPLC-ED)}

The enzymatic assay was carried out for 60 minute at $37^{\circ} \mathrm{C}$ with recombinant renalase $(100 \mu \mathrm{g} / \mathrm{mL})$ in a buffer containing $25 \mathrm{mM}$ Tris, $5 \mathrm{mM} \mathrm{NaCl}$ and $250 \mathrm{mM} \mathrm{NADH}$ $(\mathrm{pH}$ 7.5) and adrenaline, noradrenaline or dopamine as a substrate $(1 \mathrm{mM})$. The reaction was stopped by adding perchloric acid $(2 \mathrm{M})$ in an amount equal to $10 \%$ of the final reaction volume. Adrenaline, noradrenaline and dopamine levels were assayed by HPLC with electrochemical detection (HPLC-ED) (lower limit of detection of $10 \mathrm{fmol}$ of catecholamines). The substrate consumption rate was calculated by plotting substrate concentration without renalase against substrate concentration with renalase and fitting the data to the Michaelis-Menten equation by nonlinear regression (GraphPad Prism, GraphPad Software, Inc).

\section{HPLC with Photodiode Array Detection (HPLC-DAD)}

The ability of recombinant renalase to oxidize the catecholamines adrenaline, noradrenaline and dopamine to their corresponding aminochromes was examined by addition of recombinant renalase $(200 \mu \mathrm{g} / \mathrm{mL})$ to a buffer containing $25 \mathrm{mM}$ Tris, $5 \mathrm{mM} \mathrm{NaCl}$ and $250 \mathrm{mM} \mathrm{NADH}(\mathrm{pH}$ $7.5)$ adrenaline, noradrenaline or dopamine (1 mM). Protein precipitation was immediately performed by acidification with perchloric acid (10\%) followed by a rapid neutralization with $\mathrm{KHCO}_{3} 0.76 \mathrm{M}$ and centrifugation for $60 \mathrm{~s}$ at $16000 \mathrm{~g}$. Samples were immediately injected into a HPLC system (Waters model 2690) with a photodiode array detector (Waters model 996). The chromatograms were analysed as previously described [14], at $279 \mathrm{~nm}$ which corresponds to the maximum absorption wavelength of catecholamines and at $490 \mathrm{~nm}$ which corresponds to the most specific wavelength of the respective aminochromes. In a set of experiments, each catecholamine was substantially oxidized by an inorganic agent $\left(\mathrm{NaIO}_{4}\right)$ to the respective aminochromes, in order to evidence the increases of each aminochromes at 490 $\mathrm{nm}$ wavelength to determine the aminochromes evaluation time.

\section{RESULTS AND DISCUSSION}

Experimental data provide evidence that renalase exerts a hypotensive action, at least in animal models $[1,4]$. However, whether it metabolizes catecholamines remains to be 
completely proved. Any proposal on the mechanisms of actions of a newly discovered protein needs to be confirmed in the context of its functional properties.

In the present study we evaluate catecholamines degradation renalase activity by 1 ) the rate of resaruzin reduction measured by the Amplex Red Monoamine Oxidase Assay Kit; 2) the consumption of the substrate measured by HPLC$\mathrm{ED}$ and 3 ) the formation of the respective catalyse reaction end-products measured by HPLC-DAD.

In the experiments assessing renalase activity by Amplex Red Monoamine Oxidase Assay, NADH was used as a cofactor, and because it was pointed out that the copper enzyme semicarbazide-sensitive amine oxidase (SSAO) is also responsible for the degradation of catecholamines in mammalian blood plasma [5] we added semicarbazide as a specific inhibitor of SSAO, in addition to clorgyline and pargyline, as specific inhibitors of MAO-A and B, respectively.

Fig. (1) shows the renalase activity at the end of the assay, indirectly measured by the rate of resazurin reduction using the Amplex Red Monoamine Oxidase Assay Kit. The graphic shows that renalase is involved in catecholamine metabolism, being adrenaline the preferred substrate, followed by noradrenaline and dopamine.

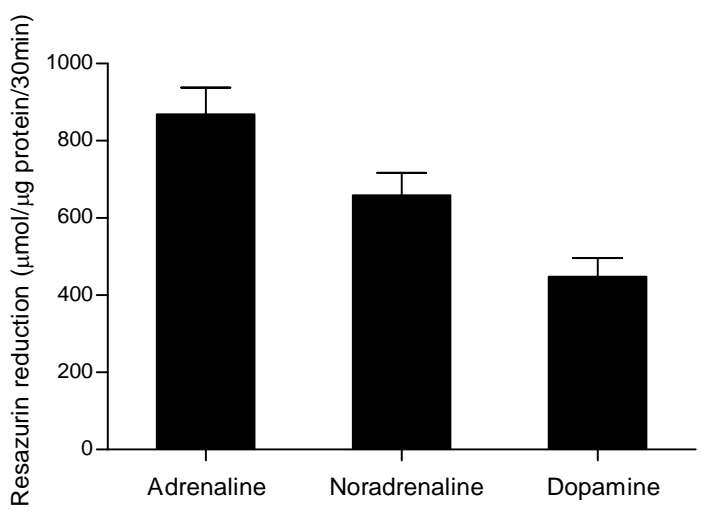

Fig. (1). Catecholamines-degrading renalase activity by the Amplex Red Monoamine Oxidase Kit. Bars are mean \pm SD of 3-4 experiments, calculated by plotting fluorescence value recorded at $30 \mathrm{~min}$ against fluorescence value recorded at $0 \mathrm{~min}$ for each condition.
Fig. (2) shows adrenaline (Fig. 2A), noradrenaline (Fig. 2B) and dopamine (Fig. 2C) consumption by recombinant renalase measured by HPLC-ED. All three catecholamines were degraded by recombinant renalase overtime confirming the data obtained with the Amplex method.

Recently it was hypothesized that aminochromes could be the end-products of the reaction catabolyzed by renalase on catecholamines [10]. These compounds have a maximum absorption at $490 \mathrm{~nm}$, and their formation can be studied by spectrophotometry [14]. Fig. (3) illustrate the chromatograms showing the formation of aminochromes resulting from the oxidation of catecholamines in the presence of recombinant renalase. In the absence of renalase, the formation of aminochromes was not detected. Also, in a control experiment carried out using recombinant renalase in the absence of catecholamines no metabolites production was detected. All three catecholamines were degraded by recombinant renalase confirming the data obtained with the other two methods above mentioned. Therefore, it was demonstrated using a validated method [14], that renalase oxidizes catecholamines to their respective aminochromes, namely adrenaline to adrenochrome, noradrenaline to noradrenochrome and dopamine to dopaminochrome.

It is known that the toxic effects induced by catecholamines oxidation processes is mainly related to production of aminochromes [15]. Aminochromes are catecholamines oxidation products that can induce oxidative stress and were described to be cytotoxic $[16,17]$. However, it seems that aminochromes, namely adrenochrome, rapidly disappears from blood by tissue uptake or by rearrangement and metabolism to adrenolutin and 5,6-dihydroxy-N-methylindone (DHMI) [18]. Although there is increasing evidence that renalase might play a role in the regulation of catecholaminergic systems by reducing circulating catecholamines, the physiological role of catecholamines renalase metabolization's end-products, the aminochromes, still unclear (Fig. 4).

In summary, these findings suggest that renalase degrades catecholamines catalyzing the formation of aminochromes. Therefore, the suggestion that recombinant renalase exerts powerful and rapid hypotensive effects on rats through circulating catecholamines degradation could be supported.

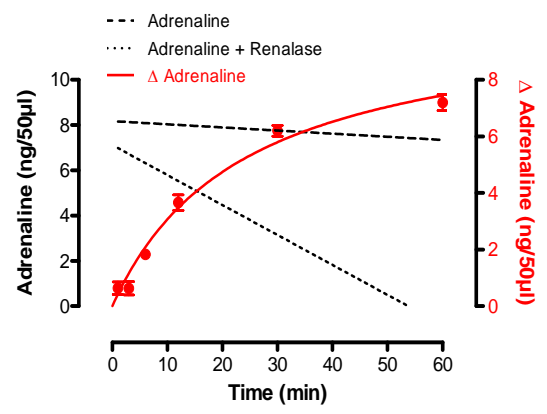

(A)

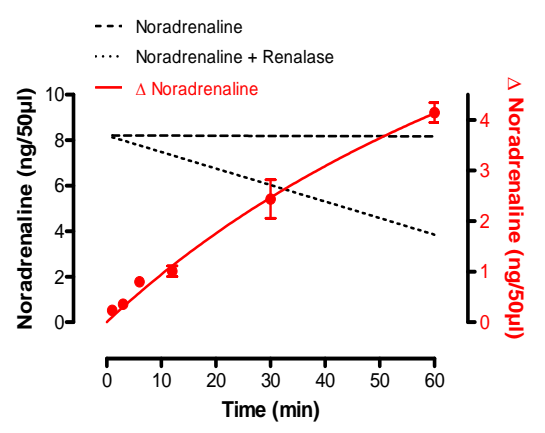

(B)

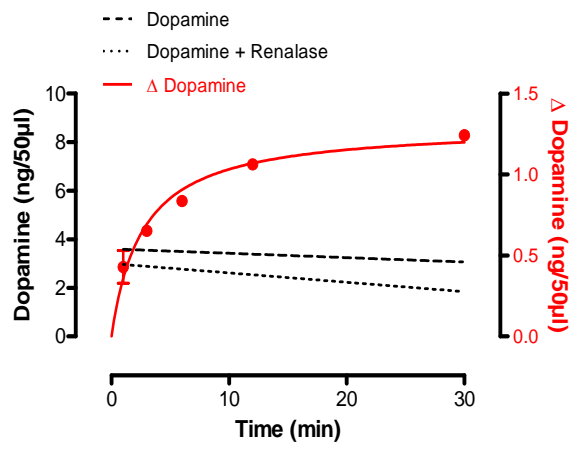

(C)

Fig. (2). Adrenaline (A), noradrenaline (B) and dopamine (C) consumption by recombinant renalase. Renalase enzymatic activity expressed as the rate of substrate consumption, calculated by the difference between catecholamine assay and catecholamine plus renalase assay. Each symbol represents the mean $\pm \mathrm{SD}$ of 3-4 experiments per concentration of substrate. 


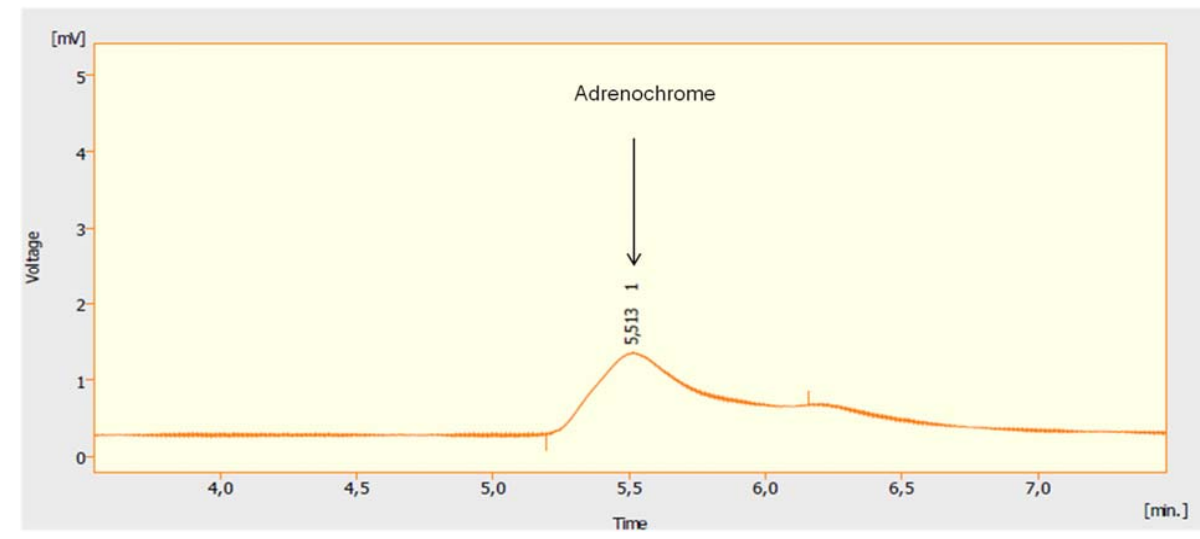

(A)

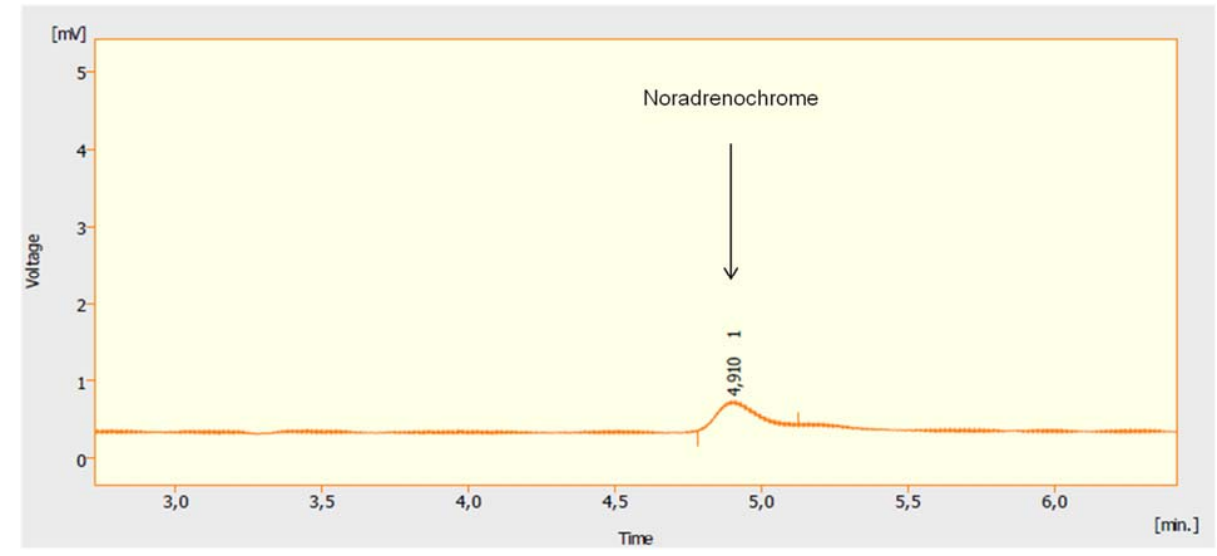

(B)

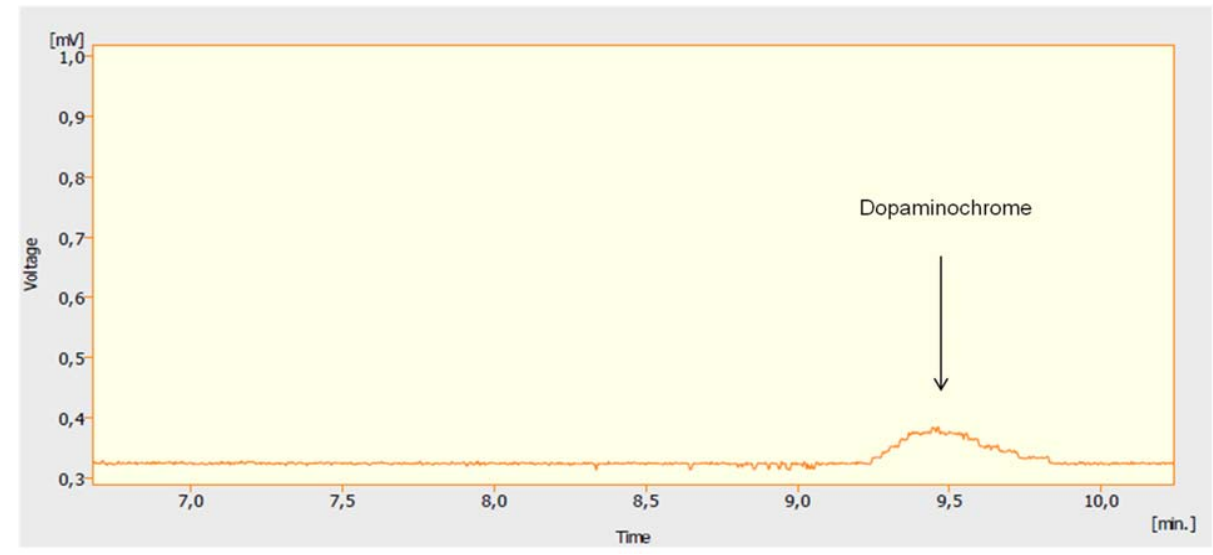

(C)

Fig. (3). Formation of aminochromes. The assay was performed in the presence of recombinant renalase and adrenaline (A), noradrenaline (B) and dopamine (C). Orange line represents the channel at $490 \mathrm{~nm}$ which corresponds to the most specific wavelength of the respective aminochromes.

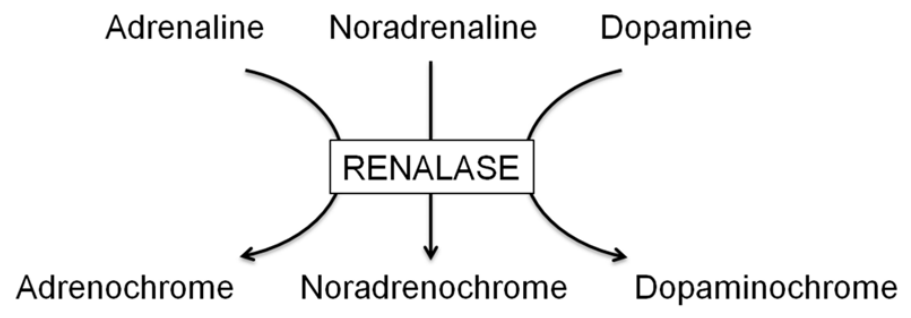

Fig. (4). Squematic representation of catecholamines metabolization to aminochromes by renalase. 


\section{CONCLUSION}

Taken together, the data presented in this study confirmed the involvement of renalase in catecholamine degradation using different technical approaches: 1) the rate of resaruzin reduction as an indirect measure of renalase oxidase activity measured by the Amplex Red Monoamine Oxidase Assay Kit; 2) the consumption of the substrate as an indirect measure of renalase activity measured by HPLC-ED and 3 ) the formation of the respective catalyse reaction endproducts as a direct measure of the action of renalase on catecholamines measured by HPLC-DAD.

\section{CONFLICT OF INTEREST}

The authors confirm that this article content has no conflict of interest.

\section{ACKNOWLEDGEMENTS}

This study was supported by grant POCI from Fundação para a FCT/COMPETE/FEDER. J. Quelhas-Santos is a PhD student supported by the fellowship SFRH/BD/39066/2007 from Fundação para a Ciência e Tecnologia/FSE.

\section{REFERENCES}

[1] Xu J, Li G, Wang P, Velazquez H, Yao X, Li Y, et al. Renalase is a novel, soluble monoamine oxidase that regulates cardiac function and blood pressure. J Clin Invest 2005 May; 115(5): 1275-80.

[2] $\mathrm{Xu} \mathrm{J}$, Desir GV. Renalase, a new renal hormone: its role in health and disease. Curr Opin Nephrol Hypertens 2007 Jul; 16(4): 373-8.

[3] Eikelis N, Hennebry SC, Lambert GW, Schlaich MP. Does renalase degrade catecholamines? Kidney Int 2011 Jun; 79(12): 1380 .

[4] Desir GV, Tang L, Wang P, Li G, Sampaio-Maia B, QuelhasSantos $\mathrm{J}$, et al. Renalase lowers ambulatory blood pressure by metabolizing circulating adrenaline. J Am Heart Assoc 2012 Aug; 1(4): e002634.

[5] Boomsma F, Tipton KF. Renalase, a catecholamine-metabolising enzyme? J Neural Transm 2007; 114(6): 775-6.
[6] Milani M, Ciriello F, Baroni S, Pandini V, Canevari G, Bolognesi $\mathrm{M}$, et al. FAD-binding site and NADP reactivity in human renalase: a new enzyme involved in blood pressure regulation. J Mol Biol 2011 Aug 12; 411(2): 463-73.

[7] Farzaneh-Far R, Desir GV, Na B, Schiller NB, Whooley MA. A functional polymorphism in renalase (Glu37Asp) is associated with cardiac hypertrophy, dysfunction, and ischemia: data from the heart and soul study. PLoS One 2010; 5(10): e13496.

[8] Desir GV, Tang L, Wang P, Li G, Sampaio-Maia B, QuelhasSantos $\mathrm{J}$, et al. Renalase lowers ambulatory blood pressure by metabolizing circulating adrenaline. J Am Heart Assoc 2012; 1(e002634)

[9] Desir G. Novel insights into the physiology of renalase and its role in hypertension and heart disease. Pediatr Nephrol 2012 May; 27(5): 719-25.

[10] Desir GV, Wang L, Peixoto AJ. Human renalase: a review of its biology, function, and implications for hypertension. J Am Soc Hypertens 2012 Nov-Dec; 6(6): 417-26.

[11] Pandini V, Ciriello F, Tedeschi G, Rossoni G, Zanetti G, Aliverti A. Synthesis of human renalase1 in Escherichia coli and its purification as a FAD-containing holoprotein. Protein Expr Purif 2010 Aug; 72(2): 244-53.

[12] Beaupre BA, Carmichael BR, Hoag MR, Shah DD, Moran GR Renalase is an alpha-NAD $(\mathrm{P}) \mathrm{H}$ oxidase/anomerase. J Am Chem Soc 2013 Sep 18; 135(37): 13980-7.

[13] Gu R, Lu W, Xie J, Bai J, Xu B. Renalase deficiency in heart failure model of rats--a potential mechanism underlying circulating norepinephrine accumulation. PLoS One 2011; 6(1): e14633.

[14] Remiao F, Milhazes N, Borges F, Carvalho F, Bastos ML, LemosAmado F, et al. Synthesis and analysis of aminochromes by HPLCphotodiode array. Adrenochrome evaluation in rat blood. Biomed Chromatogr 2003 Jan; 17(1): 6-13.

[15] Bindoli A, Rigobello MP, Galzigna L. Toxicity of aminochromes. Toxicol Lett 1989 Jul; 48(1): 3-20.

[16] Remiao F, Carvalho M, Carmo H, Carvalho F, Bastos ML. Cu2+induced isoproterenol oxidation into isoprenochrome in adult rat calcium-tolerant cardiomyocytes. Chem Res Toxicol 2002 Jun; 15(6): 861-9.

[17] Costa VM, Silva R, Ferreira LM, Branco PS, Carvalho F, Bastos $\mathrm{ML}$, et al. Oxidation process of adrenaline in freshly isolated rat cardiomyocytes: formation of adrenochrome, quinoproteins, and GSH adduct. Chem Res Toxicol 2007 Aug; 20(8): 1183-91.

[18] Noval JJ, Sohler A, Stackhouse SP, Bryan AC. Metabolism of adrenochrome in experimental animals. Biochem Pharmacol 1962 Jun; 11: 467-73.

\footnotetext{
Received: December 22, 2014

(C) Quelhas-Santos et al.; Licensee Bentham Open.
}

Revised: February 15, 2015

Accepted: February 20, 2015

This is an open access article licensed under the terms of the Creative Commons Attribution Non-Commercial License (http://creativecommons.org/licenses/by-nc/3.0/) which permits unrestricted, non-commercial use, distribution and reproduction in any medium, provided the work is properly cited. 